\title{
Picosecond Emission Dynamics of Vertical-Cavity Surface-Emitting Lasers: Spatial, Spectral, and Polarization-Resolved Characterization
}

\author{
Andreas Barchanski, Tobias Gensty, Christian Degen, Ingo Fischer, and Wolfgang Elsäßer, Senior Member, IEEE
}

\begin{abstract}
We present two-dimensional (2-D) spatially and picosecond-resolved measurements of vertical-cavity surface-emitting lasers' emission, unveiling their rich dynamics, manifesting itself in temporal changes of the modal, polarization, spectral, and spatial characteristics. The measurements providing insight into the repetitive part of the dynamics were performed with a newly developed experimental technique. In addition, we present the first 2-D, spatially resolved, single-shot event measurements which provide a full complementary picture of the dynamical behavior. The experimental results are analyzed and discussed with respect to spatio-spectral interaction mechanisms of carriers and photons, explaining the observed behavior.
\end{abstract}

Index Terms-Chaos, polarization, spatio-temporal dynamics, spectral dynamics, transverse modes, turn-on dynamics, vertical-cavity surface-emitting lasers (VCSELs).

\section{INTRODUCTION}

$\mathbf{V}$ ERTICAL-CAVITY surface-emitting lasers (VCSELs) are mature semiconductor laser structures which have undergone the development from laboratory samples to important commercial devices nowadays, and have found various areas of application, most prominently in datacom [1], [2]. They exhibit various advantages over edge emitting lasers, e.g., easy integration in two-dimensional (2-D) arrays, narrow circular output beams, on-wafer testing capability, and single-longitudinal-mode emission. The turn-on behavior of VCSELs on picosecond and nanosecond time scales plays an important role for their performance in datacom applications, in particular for the desired multigigabit per second transmission rates. Typically, aperture sizes of more than $10 \mu \mathrm{m}$ are needed to ensure sufficient output power for optical data links and optical signal processing [3]. In VCSELs with such aperture sizes, the onset of easily more than ten transverse modes can be observed, which negatively affects the desired device performance [4]-[6]. Therefore, combined spatial-spectral-temporal investigations are needed. The observation, analysis, and understanding of the spatio-temporal dynamics on picosecond timescales, associated with the multiple transverse modes, is thus not only promising for device optimization, but also allows us to gain physical insight into the underlying mechanisms and laser-matter interaction. Up to now, only spatially integrated

Manuscript received November 19, 2002; revised March 18, 2003.

A. Barchanski, T. Gensty, I. Fischer, and W. Elsäßer are with the Institute of Applied Physics, Darmstadt University of Technology, 64289 Darmstadt, Germany.

C. Degen is with Infineon Technologies AG, D-81739 München, Germany. Digital Object Identifier 10.1109/JQE.2003.813189
[7], [8] and one-dimensional cuts [9] of the time-resolved dynamics of the near-field intensity exist, precluding investigations of the dynamics of the full 2-D-spatial interactions. We have developed an experimental technique (TRIDA-Temporally Resolved Imaging by Differential Analysis) which allows us to extract the full 2-D evolution of the near-field intensity on timescales down to $10 \mathrm{ps}$. TRIDA is applicable to extract the repetitive dynamics of the laser's emission. In addition to the spatial and temporal resolution, the emission has been polarization and even spectrally resolved in order to gain insight into the different interaction mechanisms. Furthermore, this detailed information allows us to directly compare the results with theoretical modeling. The nonrepetitive part of the emission dynamics has also been investigated. We present the first 2-D spatially resolved measurements of the VCSEL's emission when pumped with single nanoseconds-long pulses, showing that the nonrepetitive part of the emission dynamics also plays an important role.

The paper is organized as follows. In Section II-A, we give detailed information on the investigated laser and the experimental setup, while Section II-B describes our TRIDA method. In Section III-A, we present time-integrated emission profiles. Section III-B concerns the polarization and spatially resolved near-field dynamics, while investigations of the combined polarization, spatially, and spectrally resolved dynamics are discussed in Section III-C. Section III-D presents measurements of single turn-on events. Section IV contains the analysis of the underlying mechanisms contributing to the observed dynamics. A summary and an outlook are presented in Section V.

\section{EXPERIMENTAL SETUP}

\section{A. Investigated VCSEL Device and Experimental Setup}

The investigated device is a selectively oxidized state-of-the-art VCSEL, with an aperture size of $14 \mu \mathrm{m}$ and a maximum optical continuous wave $(\mathrm{CW})$-output power of $3.8 \mathrm{~mW}$ at room temperature. The active medium consists of three quantum wells. The distributed Bragg reflectors are composed of 28- and 34-period layers for the p-doped top and n-doped bottom mirrors, respectively. The center emission peak wavelength, under $\mathrm{CW}$ operation amounts to $852 \mathrm{~nm}$ at an injection current of $5.3 \mathrm{~mA}$, which corresponds to about twice the threshold current $(2.3 \mathrm{~mA})$.

The experimental setup used for the spatio-temporal measurements is illustrated in Fig. 1. The VCSEL was pumped with short rectangular electrical pulses with variable width between 


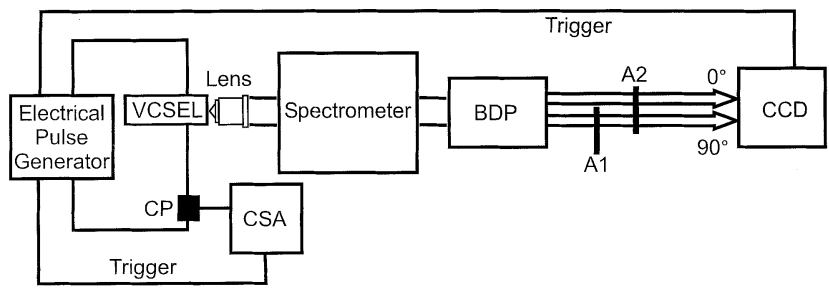

Fig. 1. Scheme of the experimental setup.

900-3000 ps and a period of $10 \mu$ s from a pulse generator. The current is detected by a current-probe $(\mathrm{CP})$ and measured with a sampling oscilloscope (CSA) with 45-GHz bandwidth. In order to allow measurements of both polarization directions simultaneously, a beam displacement prism (BDP) has been introduced into the beam, separating the laser beam into two orthogonally polarized beams with a spatial displacement of approximately $3.5 \mathrm{~mm}$. These polarization directions are aligned along the cristallographic axes, and will be referred to as the $90^{\circ} \mathrm{di}-$ rection for the polarization displayed in the horizontal direction, and $0^{\circ}$ for the one orthogonal to it. The near-field emission has been imaged onto a CCD camera, with an intensity resolution of 14 bits, by using an aspheric lens with a focal length of $4.5 \mathrm{~mm}$. For the measurements in Section III-D concerning the single events of the turn-on dynamics, an intensified CCD camera with a fast shutter time (down to $200 \mathrm{ps}$ ) and an intensity resolution of 10 bits was used. Various optical attenuators are used to prevent an over-exposure of the camera: (A1) attenuating only the $90^{\circ}$ direction which is the dominant one in the investigated device and (A2) used for both polarization directions. In Section III-C, an imaging spectrometer with a focal length of $1 \mathrm{~m}$ is used to achieve the spectral resolution, while the spatial resolution is maintained.

\section{B. TRIDA}

We start from the idea that the laser's emission dynamics can be separated into a repetitive and a nonrepetitive part. First, we concentrate on the repetitive part of the dynamics. In order to extract the repetitive part of the emission dynamics, we need to ensure that our measurements cover a statistically significant number of pulses. Therefore, the CCD-camera integrates over a constant number of pulses, by operating the pulse generator such, that it delivers a pre-selected number of pulses with a given duration and period (burst-mode). The integration in our measurements is performed over $N_{p}=36000$ pulses, in order to ensure sufficient statistics and to take full advantage of the camera's dynamical resolution.

The laser is pumped with rectangular electrical pulses with a shape, as shown schematically in Fig. 2(a)

$$
I_{\text {pump }}= \begin{cases}I_{\mathrm{on}}, & t_{0}<t \leq t_{i}, i=1,2, \ldots \\ I_{\mathrm{off}}, & \text { otherwise }\end{cases}
$$

with $t_{i}<t_{i+1}, I_{\mathrm{on}}>I_{\mathrm{thr}}$, and a pulsewidth of $t_{\text {pulse }}=t_{i}-t_{0}$. As response, the laser's emission shows the typical relaxationoscillations, as schematically depicted in Fig. 2(b). To achieve a better impedance matching, the laser is slightly pre-biased at $0.3 \mathrm{~mA}$, corresponding to $I_{\text {off }} . I_{\text {on }}$ amounts to $5.3 \mathrm{~mA}$ which is approximately twice the threshold current. The measured rise

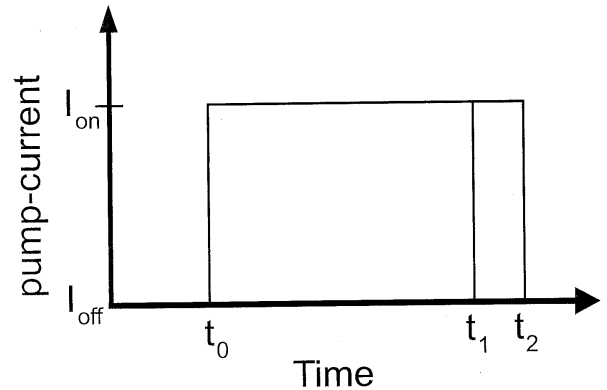

(a)

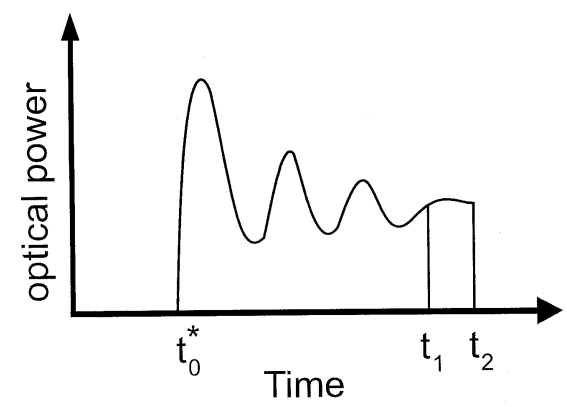

(b)

Fig. 2. Schematic depiction to illustrate the principles of TRIDA. Representation of (a) ideal rectangular electrical pulses of various length used to pump the VCSEL and (b) the resulting relaxation-oscillations. Turn-off oscillations are omitted for simplicity. In order to account for the turn-on delay, the beginning of the optical pulse is labeled as $t_{0}^{*}$.

time of the pulses amounts to approximately $500 \mathrm{ps}$. With $P(t)$ being the momentarily emitted power and $E_{I}\left(t_{i}\right)$, the integrated power, as detected by the CCD camera, one obtains

$$
\begin{gathered}
E_{I}\left(t_{i}\right)=\sum_{\substack{36000 \text { pulses } \\
i=1,2, \ldots}}\left\{\int_{t_{\text {pulse }}} P(t) d t+\int_{t_{\text {res }}} P(t) d t\right\} \\
\end{gathered}
$$

where the first integral consists of the pulses emitted by the laser and the second integral originates from spontaneous emission, due to the small bias current. This results in an intensity contribution also detected by the camera within the time between the pulses $\left(t_{\text {res }}\right)$, because the shutter of the camera remains open until all 36000 pulses are accumulated. However, the value of the second integral is negligible as compared to the first.

In order to obtain the momentarily emitted power $\tilde{P}(t)$, one has to form the differential quotient at the position $t_{i+1}$

$$
\tilde{P}\left(t_{i+1}\right)=\lim _{t_{i} \rightarrow t_{i+1}} \frac{1}{N_{p}} \cdot \frac{E_{I}\left(t_{i+1}\right)-E_{I}\left(t_{i}\right)}{t_{i+1}-t_{i}} .
$$

The smallest experimentally possible time step from $t_{i}$ to $t_{i+1}$ amounts to $10 \mathrm{ps}$, and is limited by the pulse generators time resolution. We approximate the differential quotient of the momentarily emitted power by the difference quotient. The denominator of (3) only provides a constant factor to the difference quotient. Because $t_{i+1}-t_{i}=10 \mathrm{ps}=$ const., it is sufficient to subtract the intensities $E_{I}$ of two consecutive images. For a position in the near-field emission of the laser with the coordinates $(x, y)$, the general form of the equation used can be extended to

$$
\tilde{P}\left(x, y, t_{i+1}\right) \propto E_{I}\left(x, y, t_{i+1}\right)-E_{I}\left(x, y, t_{i}\right) .
$$




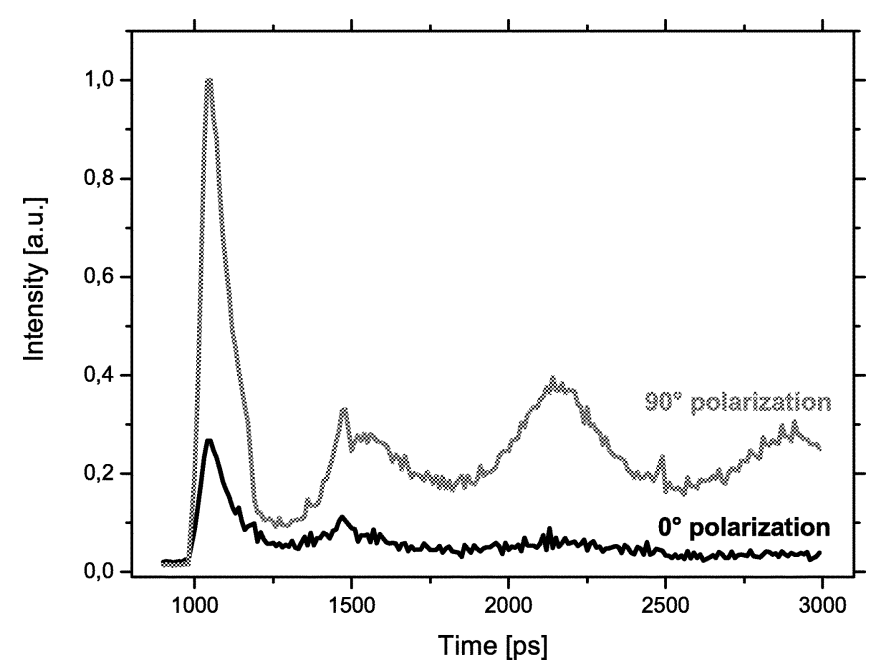

Fig. 3. Evolution of the relaxation oscillations extracted from the differential images. The gray curve represents the $90^{\circ}$ polarization direction, while the black curve depicts the evolution of the $0^{\circ}$ polarization direction. The turn-on time of the electrical pulse $t_{0}$ was chosen to be zero.

In order to prove that the TRIDA method gives reliable results, it has to be verified whether the extracted momentary power $\tilde{P}(t)$ exhibits the expected, well-known relaxation oscillation behavior, as theoretically derived from the rate equations, and as already schematically depicted in Fig. 2(b). We extracted the evolution of the relaxation oscillation by summing up the intensity of all pixels of a differential image, and plotting it versus time. This is depicted in Fig. 3 for both polarization directions. From the excellent agreement with the expected relaxation-oscillation behavior, we conclude that the turn-on jitter has no significant influence on our measurements [6], [10]. Furthermore, the smoothness of the curves in Fig. 3 confirm well that TRIDA gives reliable data, with a reasonable signal-to-noise ratio. A third proof for a sufficient signal-to-noise ratio can be given by comparing different images recorded under same conditions. The differential images originating from emission under unchanged conditions only show very little difference in the near-field emission intensity compared to the differences from consecutive images. Now that we have demonstrated the principles and the reliability of TRIDA, the obtained results will be presented in the next section.

\section{EXPERIMENTAL RESULTS}

We begin with the presentation of the near-field emission intensity images.

\section{A. Time Integrated Images}

In order to give an overall picture of the spatially resolved emission behavior, we start by presenting time-integrated images of the VCSEL's near-field emission intensity. Those kinds of near-field emission profiles are typically found under $\mathrm{CW}$ and quasi-CW operation by means of CCD-camera measurements with standard exposure times [4], [5]. The temporally integrated near-field intensity is plotted in Fig. 4. The left side of the image represents the $0^{\circ}$ polarization direction, while the right side shows the $90^{\circ}$ polarization direction. A pulsewidth of $3 \mathrm{~ns}$, an injection current of $5.3 \mathrm{~mA}$, a period of $10 \mu \mathrm{s}$, and

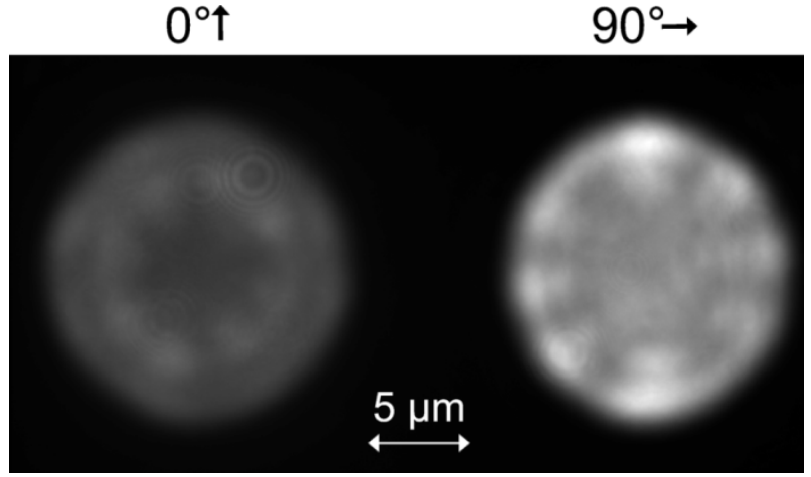

Fig. 4. Time-integrated near-field emission of the investigated device. A pulsewidth of $3 \mathrm{~ns}$ and pumping conditions described in the text were used. The images show the near-field intensity after integration over 36000 pulses. The left part represents the $0^{\circ}$ polarization direction, while the right part shows the $90^{\circ}$ polarization direction.

36000 pulses were used for this exposure. A period of $10 \mu \mathrm{s}$ prevents the occurrence of thermal effects [11], [12]. The time integrated images depict the complex structure of the emission profile of the examined device, consisting of numerous transverse modes. In terms of the nomenclature used in (2), Fig. 4 depicts $E_{I}(3 \mathrm{~ns})$. From these kinds of images the temporally resolved images have been obtained by TRIDA.

\section{B. Polarization and Time-Resolved Near-Field Dynamics}

In this section, we analyze the turn-on behavior during the first relaxation oscillation peak, as well as the subsequent emission dynamics with the help of the TRIDA method. A pump current of $5.3 \mathrm{~mA}$ (equals 2.3 times threshold), and pulsewidths from 900 to $3000 \mathrm{ps}$ have been used. The obtained spatially integrated behavior, i.e., the polarization-resolved relaxation oscillations, have been already plotted in Fig. 3. The gray curve depicts the evolution of the optical power of the $90^{\circ}$ polarization direction, the black one of the $0^{\circ}$ polarization direction. The investigated device exhibits an onset of laser emission in the $90^{\circ}$ direction, $1000 \mathrm{ps}$ after the start of the electrical pulse, the $0^{\circ}$ direction lags about 20 ps. During the first 150 ps of the emission, the laser exhibits the first relaxation oscillation peak which we analyze in the following in detail. For a better visualization, every differential snapshot of the laser's near-field intensity has been individually normalized to its maximum intensity after the subtraction. This allows us to pursue the qualitative changes of the emission, but not to compare the absolute intensity among the images.

Fig. 5 shows the evolution of the VCSEL's near-field intensity during the first relaxation oscillation peak. In agreement with the measurements shown in Fig. 3, the $0^{\circ}$ polarization direction lags the $90^{\circ}$ polarization direction which can be seen in the intensity distribution of the 1000 -ps image. Here, the $90^{\circ}$ polarization direction already shows laser emission, while the $0^{\circ}$ polarization direction just begins to form a mode profile from spontaneous emission. The following snapshots at 1030 and 1060 ps illustrate that the laser turns on in a superposition of low order modes, adding up to the so-called doughnut shape. However, the vertical mode in the $90^{\circ}$ polarization direction emits more power, in particular, in two bright spots at the top and bottom part of the doughnut. The intensity increase in the $0^{\circ}$ 


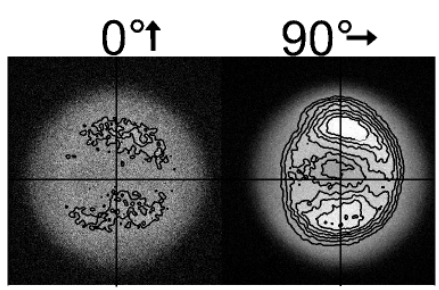

$1000 \mathrm{ps}$

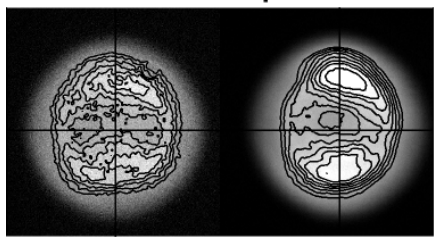

1030ps

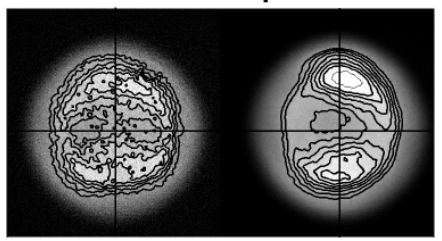

1060ps

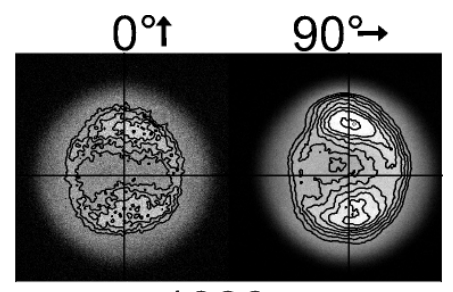

1090ps

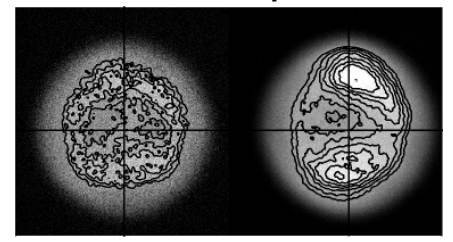

$1100 \mathrm{ps}$

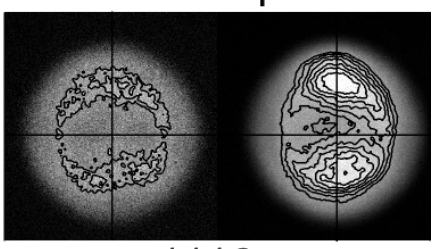

1110ps

Fig. 5. Evolution of the VCSELs near-field intensity during the first relaxation oscillation peak. Below every snapshot the temporal position of the differential image is given in picoseconds. A linear grayscale encoding was used. A snapshot represents the increase in near-field intensity during $10 \mathrm{ps}$, with white being the maximal intensity increase. The left part of each image shows the $0^{\circ}$ polarization direction, the right part the (dominant) $90^{\circ}$ polarization direction For eye guidance, thin black crosshairs and a contour-plot have been overlaid.

polarization direction is nearly uniformly distributed over the doughnut ring. A slight rotation in the near-field emission intensity of the dominant polarization direction can be observed, when analyzing the snapshots at 1090,1100 , and $1110 \mathrm{ps}$. The bright spot in the lower part of the doughnut has moved a few degrees clockwise from the intensity distribution at $1090 \mathrm{ps}$ to the distribution at $1100 \mathrm{ps}$. In the following step from 1100 to $1110 \mathrm{ps}$ the rotation direction is reversed, and the spot moves to its former position at $1090 \mathrm{ps}$. This indicates a small rotational flicker in the emission. Except for this rotation, the intensity distribution remains nearly unchanged during the first relaxation oscillation peak. This changes drastically, when analyzing the subsequent evolution of the near-field emission intensity. We find that higher order modes are excited, resulting in a distinct emission dynamics of the VCSELs.

Fig. 6 depicts four snapshots of these near-field emission intensity changes from 1720 to $1750 \mathrm{ps}$, namely between the second and third relaxation oscillations peak. The images give evidence for a rich dynamical behavior in the emission profile in both polarization directions. At $1720 \mathrm{ps}$, in the $0^{\circ}$ polarization direction the center of the aperture is filled with a bright spot of intensity increase, along with the also bright horizontal line, having approximately the same width as the spot. In the same polarization direction, $10 \mathrm{ps}$ later, the center of the aperture remains mostly dark. The previously mentioned horizontal line through the near-field emission intensity carries substantially less intensity than its peripheral surroundings. Another $10 \mathrm{ps}$ later, $1740 \mathrm{ps}$ after the beginning of the electrical pulse, the intensity change is nearly uniform over the whole aperture, having
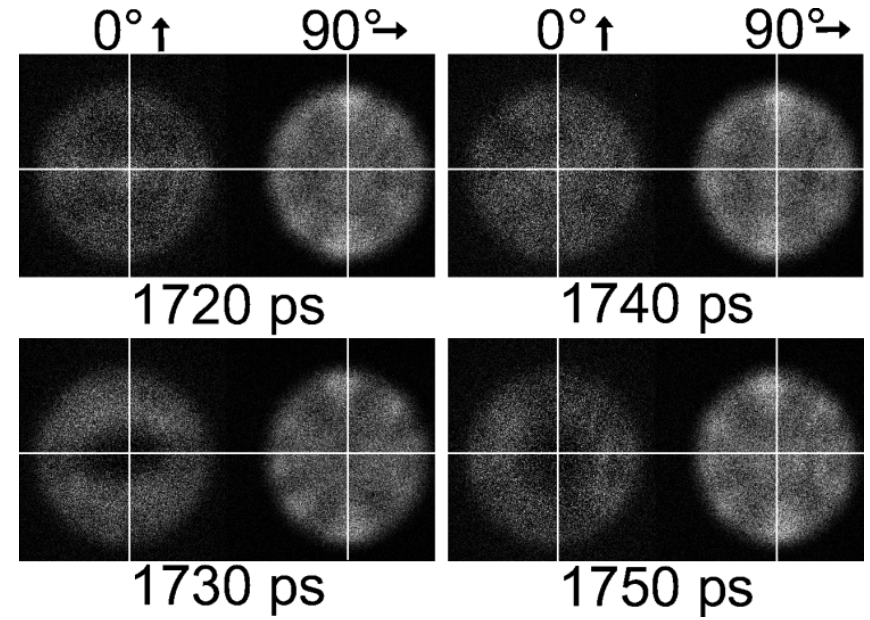

Fig. 6. Evolution of the VCSEL's near-field intensity after the second relaxation oscillation peak. Below every snapshot, the temporal position of the differential image is given in picoseconds. A linear grayscale encoding was used. A snapshot represents the change in output power during $10 \mathrm{ps,} \mathrm{with}$ white being the maximal power increase. The left part of each image shows the $0^{\circ}$ polarization direction, the right part the (dominant) $90^{\circ}$ polarization direction. For eye guidance, thin white crosshairs have been overlaid.
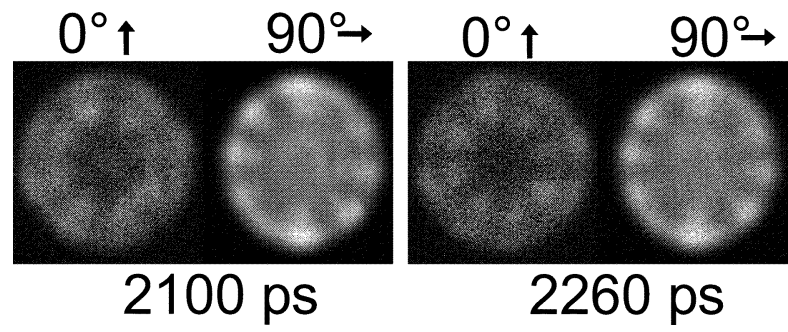

Fig. 7. Snapshots of the VCSEL's near-field intensity change within the third relaxation-oscillation peak. Below every snapshot, the temporal position of the differential image is given in picoseconds. Here, a snapshot represents the change in output power during $20 \mathrm{ps}$, with white being the maximal power increase. The same linear grayscale encoding which was used in the previous figures was applied. The left part of each image shows the $0^{\circ}$ polarization, the right part the (dominant) $90^{\circ}$ polarization direction.

four small dark points aligned symmetrically in the outer area of the aperture. The dark spot in the center has vanished, and the center shows an intensity increase, again. The last image in this row was taken at $1750 \mathrm{ps}$. As in the snapshot at $1730 \mathrm{ps}$, the center of the aperture remains dark, indicating that during the last $10 \mathrm{ps}$ there was no intensity increase in this area. Furthermore, a dark line similar to the one in the 1730 ps image, can also be seen, but this time it is aligned nearly vertical, with an approximately $5^{\circ}$ counterclockwise rotation. The $90^{\circ}$ polarization direction also exhibits an interesting dynamical behavior. The bright line of intensity increase from the top to the bottom of the aperture rotates between two positions, by approximately $5^{\circ}$ counterclockwise and $5^{\circ}$ clockwise, respectively. This kind of rotational flicker was already observed in Fig. 5 during the first relaxation oscillation peak. A discussion of the underlying mechanisms will be given in Section IV.

Fig. 7 shows two snapshots of the intensity increase, taken at the third relaxation oscillation peak at 2100 and $2260 \mathrm{ps}$, respectively. The time resolution in this figure was changed to 20 ps, in order to have a better signal-to-noise-ratio and, therefore, to better illustrate the complementary behavior. In both images 


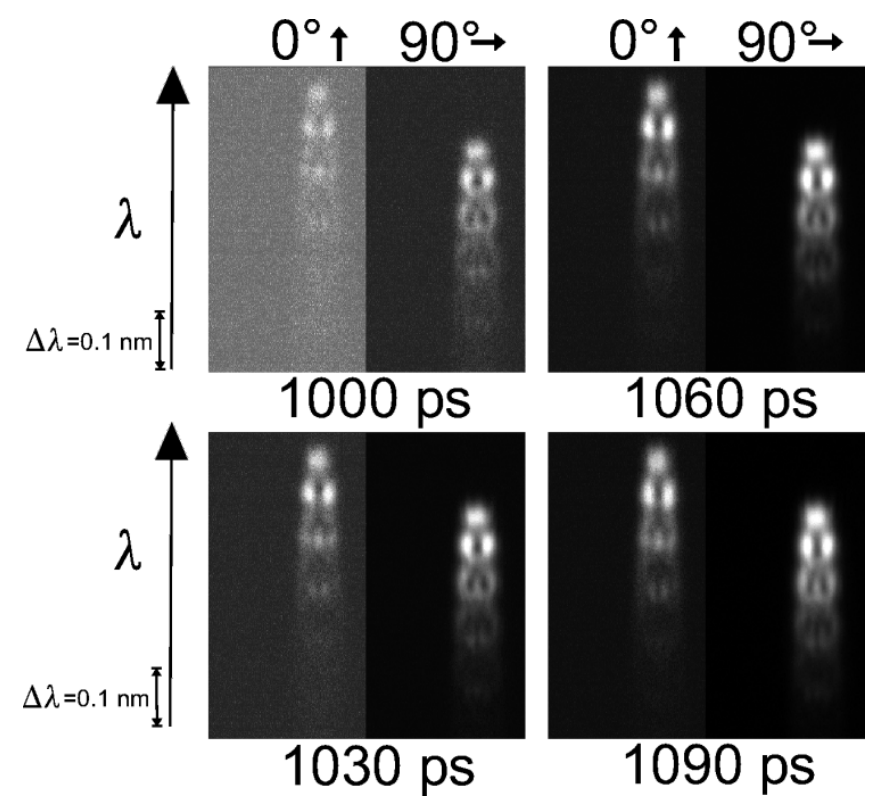

Fig. 8. Evolution of the spatially, spectrally, and polarization resolved VCSEL's near-field emission intensity during the first relaxation oscillation peak. Below every snapshot, the temporal position of the differential image is given in picoseconds. A linear grayscale encoding was used. Each snapshot represents the change in output power during $10 \mathrm{ps}$, with white being the maximal power increase. The left part of each image shows the $0^{\circ}$ polarization direction, the right part the (dominant) $90^{\circ}$ polarization direction. The wavelength increases from bottom to top of each snapshot.

the center of the $0^{\circ}$ polarization direction remains dark, contrary to the $90^{\circ}$ polarization direction which shows a bright spot at this place. The peripheral area of the aperture shows eight intensity maxima in both polarization directions. However, they are offset in angle against each other, in a way that a maximum of the intensity increase of the $0^{\circ}$ polarization direction can be found at locations where the $90^{\circ}$ polarization direction shows a minimum of the intensity increase, and vice versa. This complementary behavior can be observed in most snapshots after the first relaxation oscillation peak. Both images show a significant similarity, that can be observed in nearly all images after 2000 ps. At this times, the near-field emission intensity is composed of a superposition of several modes which does not change its appearance, the repetitive part of the dynamical behavior consists of an angular rotation of the bright spots, as previously described. However, we would like to emphasize that this does not exclude the appearance of the nonrepetitive part of the emission dynamics which can not be measured with TRIDA.

\section{Polarization, Time, and Spectrally Resolved Near-Field Dynamics}

Every transverse mode in a VCSEL corresponds to a different wavelength. Therefore, by spectrally resolving the near-field emission intensity, it is possible to investigate the dynamics of each mode separately. In this subsection, we present near-field emission intensity images which have been furthermore spectrally resolved. By using an imaging spectrometer, we have succeeded in spatially separating the modes maintaining their spatial structure, which will allow us to investigate spectral interaction mechanisms. Fig. 8 depicts a combination of the

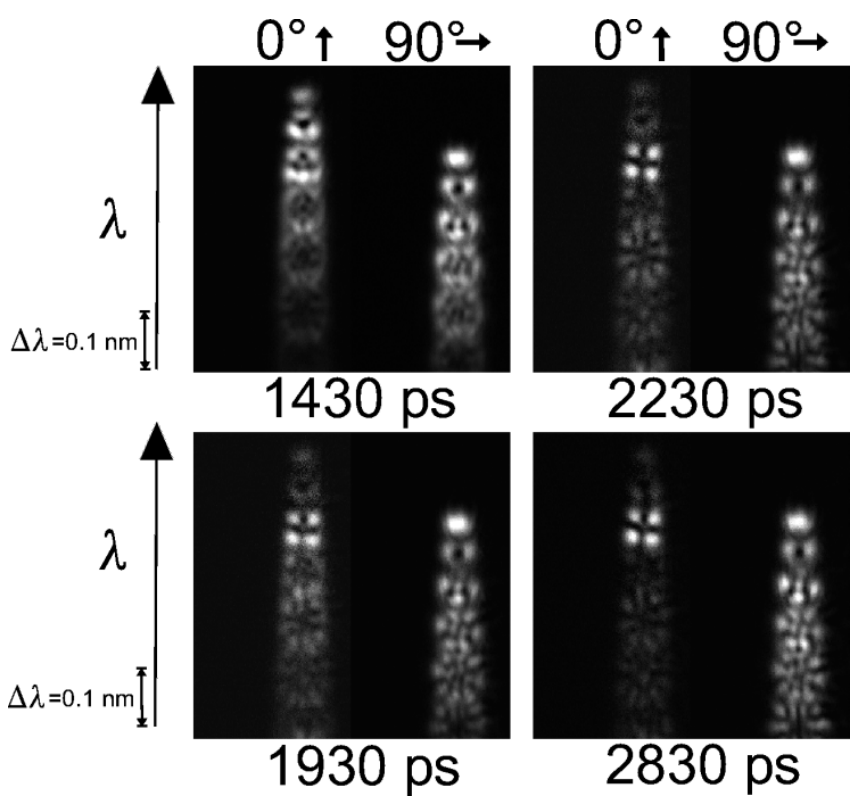

Fig. 9. Evolution of the spatially, spectrally and polarization resolved VCSEL's near-field emission intensity after the first relaxation oscillation peak. Below every snapshot, the temporal position of the differential image is given in picoseconds. In these images, a snapshot represents the change in output power during $100 \mathrm{ps}$, with white being the maximal power increase. A linear grayscale encoding was used. The left part of each image shows the $0^{\circ}$ polarization direction, the right part the (dominant) $90^{\circ}$ polarization direction. The wavelength increases from bottom to top of each snapshot.

spatially, spectrally, and polarization resolved evolution of the VCSEL's near-field during the first relaxation oscillation peak. The horizontal axis represents the spatial coordinate, the vertical axis is a combination of both spatial and spectral coordinates, with the wavelength increasing from the bottom to top of each snapshot [13], [14]. As a consequence, spatially extended transverse modes that are only slightly separated in wavelength may overlap in this representation. The beam-displacement prism has been carefully aligned to prevent wavelength offset among both polarization directions. The large birefringence splitting which is the spectral spacing among the fundamental Gaussian modes in orthogonal polarization directions is quite noticeable. A rough estimation of the birefringence splitting, performed with an optical spectrum analyzer having a maximal resolution of $0.05 \mathrm{~nm}$, provides a value of approximately 0.11 $\mathrm{nm}$, or $50 \mathrm{GHz}$, respectively. The spectrally resolved images are in correspondence with the results obtained in the analysis of Fig. 5. The $0^{\circ}$ polarization direction at 1000 ps in Fig. 8 has still a low intensity which can be seen by the low contrast to the gray background originating from the normalization of each image. During the first relaxation oscillation peak, only modes of lower order are excited in the device, as depicted by Fig. 8 . In both polarization directions, the third order is the highest order of noticeable intensity increase. The relative intensity increase among the contributing modes during the first relaxation oscillation peak is negligible.

Fig. 9 presents spatially, spectrally, and polarization-resolved snapshots taken after the first relaxation oscillation peak, demonstrating the subsequent spectral and spatial evolution. The snapshots show the intensity change during $100 \mathrm{ps}$. In this case, it has been necessary to reduce the time resolution, in 
order to obtain a better signal-to-noise ratio for the interpreta-

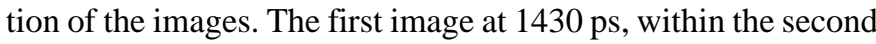
relaxation oscillation peak, already shows two more mode orders than during the first relaxation-oscillation peak. At 1930 ps, 500 ps later, there are even more modes excited than shown in the figure, because some have been cut due to the limited size of the CCD chip. Under these conditions, about ten modes occur in the laser's near-field intensity. While in the (dominant) $90^{\circ}$ polarization direction, the relative intensity increase among the modes mostly remains uniform, there is a drastic change in the modal behavior in the $0^{\circ}$ polarization direction. Throughout the evolution of the pulse, one four-lobed mode remains with a relative maximum intensity increase, while all other modes in the $0^{\circ}$ polarization direction show a relative smaller intensity increase. The fundamental Gaussian mode in the $90^{\circ}$ polarization direction is spectrally aligned at the same position as the bright four-lobed mode in the $0^{\circ}$ polarization direction which is of the second order. This spectral alignment implies the importance of spectral interactions, and will be a point in the forthcoming discussion.

\section{Nonrepetitive Near-Field Dynamics}

In the previous subsections, we succeeded in resolving the repetitive dynamics of the VCSEL's emission, with the help of TRIDA. An integration over 36000 pulses has been performed, in order to obtain statistically significant results. Whether there is also a significant nonrepetitive part in the emission dynamics of VCSELs has been unclear. Some numerical modeling indicates that this could be indeed the case [15], [16]. Here, we therefore perform first 2-D, spatially resolved single-shot event measurements. They allow us to investigate the nonrepetitive part of the near-field emission intensity, therefore providing a complementary and complete picture of the dynamics. In recent years, cameras with very fast shutter times, down to $200 \mathrm{ps}$, became available. With the further assistance of intensifying components, it became possible to detect very low intensities, and even single photons, with a 2-D spatial localization [17]. Only with the help of these advances in imaging technology did the forthcoming measurements become possible.

Here, we present measurements of the near-field intensity distribution performed with low exposure times, using the previously explained setup. Four snapshots of a single-shot event are depicted in Fig. 10. Each image shows the near-field intensity distribution of the VCSEL's emission when pumped with an electrical pulse of 3-ns width and a current of $5.3 \mathrm{~mA}$. The pumping conditions are equal to those used in Fig. 4; however, we point out that Fig. 4 depicts the time integrated intensity of 36000 pulses, while the snapshots in Fig. 10 represent the emission when pumped with a single pulse. The left part of each image shows the $0^{\circ}$ polarization direction, the right part the (dominant) $90^{\circ}$ polarization direction. First, summing up the intensity distribution of a large amount of single-shot snapshots, we obtain the same near-field intensity distribution profiles as in the time integrated measurements that were used for TRIDA, confirming the excellent reliability of our measurements. Second, Fig. 10 shows that the intensity distribution in each pulse shows similar features, like the appearance of intensity maxima in the periphery of the aperture. Third, in addition
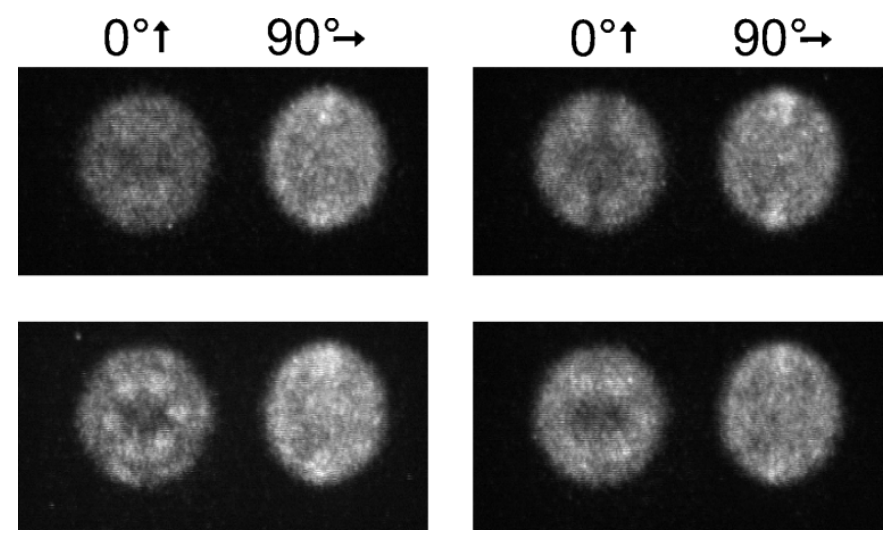

Fig. 10. Single-shot images of the VSCEL near-field emission intensity. Each image represents one 3-ns pulse at the same pumping condition as before. The left part of each image shows the $0^{\circ}$ polarization direction, the right part the (dominant) $90^{\circ}$ polarization direction.

to the similar features of each pulse, distinct differences in the intensity distribution of different pulses are identifiable. Here, we would like to concentrate only on the differences among the single-shot events, although no obvious parameters were changed between each exposure. The $0^{\circ}$ polarization direction in the upper left image shows a nearly uniform distribution of the emitted intensity over the whole aperture, while the other three snapshots have a dark spot in the center of the aperture. The upper right image shows a dark vertical line through the aperture in the near-field emission intensity of the $0^{\circ}$ polarization direction, which can not be found in the other images. The bottom left image exhibits six clearly visible intensity maxima in the $0^{\circ}$ polarization direction, indicating, that a mode of third order exhibits a maximum intensity. These first two-dimensionally and spatially resolved images allow us, for the first time, to give evidence for a significant nonrepetitive part in the turn-on dynamics of VCSELs.

\section{ANALYSIS OF THE UNDERLYING MECHANISMS}

In this section, a qualitative analysis of the physical mechanisms contributing to the behavior depicted in Section III will be performed. Our measurements present for the first time a spatial 2-D resolution at time scales down to 10 ps. Furthermore, polarization and even spectral resolution have been obtained. Our analysis will begin with a discussion of the modal behavior during the first relaxation-oscillation peak, followed by an investigation of spatial mechanisms. The analysis is extended by a discussion of spectral interactions. The section closes with a discussion of the nonrepetitive emission dynamics.

We find that the laser turns on in modes of low order, before in the further time evolution higher order modes begin to contribute to the emitted intensity. Modeling results concerning the turn-on behavior also indicate that the laser starts to emit in low order modes due to their lower threshold, compared with higher order modes [15]. Spectrally, however, not spatially resolved experiments performed by Buccafusca et al. [7], [8], [19] agree very well with the observed behavior. The set of excited modes during the first relaxation oscillation peak depends on pumping conditions, and there is little change in the relative intensity increase among the modes, indicating that during this 
time the threshold behavior of each mode dominates the evolution of the near-field intensity [8]. The appearance of higher order modes and their dynamical behavior has been experimentally shown by Giudici et al. with the help of a streak camera [9]. These near-field emission intensity images obtained by a streak camera are limited to a spatially one dimensional resolution, allowing to detect the dynamical spectral evolution, however not to analyze interaction between modes and other spatial mechanisms that are theoretically supposed to play an important role in the modal distribution and the emission dynamic [18], [20].

The dynamical behavior following the first relaxation oscillation peak is a result of various spatial interactions taking place in the device. The complementarity between both polarization directions, shown in Fig. 7, originates from a competition of both polarization directions for the available gain in the active medium. Among the polarization directions, transverse modes with a minimum spatial overlap are favored. Theoretical calculations also predict this behavior [15], [21]. From our experimental results, not only can the spatial interactions between the polarization directions be identified, but also a dynamical behavior inside each polarization direction can be analyzed in terms of spatial mechanisms. The beginning rotation of the intensity increase in the last three images of Fig. 5 already gives an idea of the importance of spatial hole burning. Fig. 6 clearly demonstrates how spatial hole burning affects the distribution of the intensity increase following the first relaxation oscillation peak, although the spatially integrated power only shows a slight change during this time. The time evolution of the intensity increase, as depicted in Fig. 6, can be attributed to modal competition due to spatial hole burning. As the carrier density exceeds threshold, a mode is excited and begins to contribute to the emission which can be seen at 1720 ps in the lowest order mode in the $0^{\circ}$ polarization direction. The light-matter interaction now reduces the carrier density in the modal emission area, and reduces the gain of the mode, possibly even below threshold resulting in extinction, as illustrated $10 \mathrm{ps}$ later. At this point, the carrier concentration can again increase in the modal emission area, leading to higher gain, and a possible new turn-on of the mode, as it is obvious at $1740 \mathrm{ps}$. The behavior can be observed several times. In the $90^{\circ}$ polarization direction, the effect is harder to be recognized because of the higher intensity of high order modes. However, the noticeable bright vertical line through the aperture rotates slightly a few degrees to the left and right, showing a behavior that can be explained in analogy with the $0^{\circ}$ polarization direction, as a result of spatial hole burning. Spatial hole burning also affects the intensity distribution among different modes. After burning a hole in the carrier density, it is possible that the changed carrier distribution (spatially) favors the onset of other transverse modes. This also results in a distinct dynamical behavior.

The spectrally resolved images presented in Section III-C allow a characterization of both spectral and spatial interactions. The evolution of the spectrally resolved emission of the $0^{\circ}$ polarization direction in Fig. 9 shows that after about 2000 ps, one four-lobed mode exhibits the maximal intensity increase which is spectrally aligned at the same position as the fundamental Gaussian mode in the $90^{\circ}$ polarization direction. Under the operating conditions used, the gain of the active medium exhibits its maximum at this spectral position, resulting in the amplification of the described modes [12]. The spatial intensity distribution can be explained with the competition of the modes for the available gain. The fundamental mode, observed in the $90^{\circ}$ polarization direction, has its intensity concentrated in the center of the aperture. In contrast, the intensity of the four-lobed mode is concentrated in the periphery of the aperture, resulting in a minimal spatial overlap of both modes. This behavior clearly confirms the preceding analysis in this section.

The images in Section III-D clearly indicate that nonrepetitive dynamics also play an important role in the VCSEL's emission, as was already mentioned in Section II-B. This nonrepetitive part can be either due to the influence of spontaneous emission in the buildup of every turn-on pulse, or due to the onset of chaotic dynamics in these devices. Numerical results predict the importance of nonrepetitive dynamics, and even chaotic emission due to nonlinear spatio-temporal interaction of laser emission and active medium [15]. Our experimental findings of the small turn-on jitter, and furthermore, the retarded onset of the evolution of the spatio-temporal dynamics several tens of picoseconds after the onset of the laser emission indicate that spatio-temporal chaos can indeed be found in the VCSEL's emission. Therefore, we attribute the nonrepetitive part of the VCSEL's emission dynamics to local spatio-temporal interactions like spatial hole burning in the active medium. In numerical modeling, the resulting dynamical phenomena have been found to go even beyond a modal description, requiring a full spatio-temporal treatment [22]. Similar nonlinear dynamical behavior has already been found and well investigated in the field of broad-area semiconductor lasers [23]. Further detailed investigations to classify the role of spatio-temporal chaos in the emission of multimode VCSELs are under way. Finally, it should be mentioned that current modulation with datacom-typical pulse-repetition rates in the gigahertz range, therefore being much higher than in our experiments, can lead to additional chaotic emission phenomena [16], underlining the importance to consider and study chaotic emission in VCSELs.

\section{Summary AND OUtLOOK}

We have developed TRIDA (Temporal Resolved Imaging by Differential Analysis), a new method to analyze the repetitive part of the turn-on behavior, and the subsequent dynamical evolution of a VCSEL's near-field emission intensity on a 10-ps time scale with polarization, spectral, and spatial 2-D resolution. With the help of TRIDA, we were able to obtain detailed information about spatio-temporal interactions in VCSELs. We have shown that the analyzed device turns on in a few low-order transverse modes, determined by the pumping conditions. The relative intensity increase distribution remains nearly constant during the first relaxation oscillation peak. The subsequent emission is characterized by a distinct dynamical behavior, originating from various spectral and spatial interaction mechanisms. Spatial hole burning has been identified as a very important effect contributing to the observed dynamics on the examined time scales. Furthermore, from the observed spectral behavior we were able to identify the role of the gain maximum of the active medium. The first spatially resolved, 
single-shot event measurements presented in Section III-D have shown that there is also a nonrepetitive contribution to the near-field emission, probably even chaotic dynamics, in agreement with numerical simulations [15].

Further research should include the investigation of detailed parameter dependencies like injection current or temperature on the turn-on emission dynamics. TRIDA could of course also be applied to investigate other VCSEL structures. Also, the nonrepetitive dynamics deserve further attention. It is an open question whether the observed nonrepetitive part of the dynamics is a transient effect or prevails even for $\mathrm{CW}$ operation. The dynamics of VCSELs will remain an interesting research field, providing further insight into the physics of semiconductor lasers and promising device optimization.

\section{ACKNOWLEDGMENT}

The authors would like to thank Infineon Technologies AG for providing them with the excellent VCSEL structures.

\section{REFERENCES}

[1] P. Schnitzler, U. Fiedler, G. Reiner, B. Weigl, W. Zick, and K. J. Ebeling, "Bias-free $1-\mathrm{Gb} / \mathrm{s}$ data transmission using top vertical-cavity surfaceemitting laser diodes," IEEE Photon. Technol. Lett., vol. 9, pp. 693-695, May 1997.

[2] K. J. Ebeling, U. Fiedler, R. Michalzik, G. Reiner, and B. Weigl, "Efficient vertical cavity surface emitting laser diodes for high bit rate optical data transmission," AEU Int. J. Electron. Commun., vol. 50, pp. 316-326, June 1996.

[3] U. Fiedler, G. Reiner, P. Schnitzer, and K. J. Ebeling, "Top surface-emitting verical-cavity laser diodes for $10-\mathrm{Gb} / \mathrm{s}$ data transmission," IEEE Photon. Technol. Lett., vol. 8, pp. 746-748, Jun. 1996.

[4] H. Li, T. L. Lucas, J. G. McInerney, and R. A. Morgan, "Transverse modes and patterns of electrically pumped vertical-cavity surface-emitting semiconductor lasers," Chaos, Solitons \& Fractals, vol. 4, pp. 1619-1636, 1994.

[5] C. Degen, I. Fischer, and W. Elsäßer, "Transverse modes in oxide confined VCSELs: Influence of pump profile, spatial hole burning and thermal effects," Opt. Expr., vol. 5, pp. 38-46, Aug. 1999.

[6] J. Dellunde, M. C. Torrent, J. M. Sancho, and K. A. Shore, "Statistics of transverse mode turn-on dynamics in VCSEL's," IEEE J. Quantum Electron., vol. 33, pp. 1197-1204, July 1997.

[7] O. Buccafusca, J. L. A. Chilla, J. J. Rocca, C. Wilmsen, S. Feld, V. Morozov, and R. Leibenguth, "Transverse mode dynamics in vertical cavity surface emitting lasers excited by fast electrical pulses," Appl. Phys. Lett., vol. 68, pp. 590-592, Jan. 1996.

[8] O. Buccafusca, J. L. A. Chilla, J. J. Rocca, P. Brusenbach, and J. MartinRegalado, "Transient response of vertical-cavity surface-emitting lasers of different active-region diameters," IEEE J. Quantum Electron., vol. 35, pp. 608-615, Apr. 1999

[9] M. Giudici, J. R. Tredicce, G. Vaschenko, J. J. Rocca, and C. S. Menoni, "Spatio-temporal dynamics in vertical cavity surface emitting lasers excited by fast electrical pulses," Opt. Commun., vol. 158, pp. 313-321, Dec. 1998.

[10] L. Zei, K. Obermann, T. Czogalla, and K. Petermann, "Turn-on jitter of zero-biased nearly single-mode VCSEL's," IEEE Photon. Technol. Lett., vol. 11, pp. 6-8, Jan. 1999.

[11] C. Degen, I. Fischer, and W. Elsäßer, "Thermally induced local gain suppression in vertical cavity surface emitting lasers," Appl. Phys. Lett., vol. 76, pp. 3352-3354, June 2000.

[12] C. Degen, I. Fischer, W. Elsäßer, L. Fratta, P. Debernardi, G. Bava, M Brunner, R. Hvel, M. Moser, and K. Gulden, "Transverse modes in thermally detuned oxide confined vertical cavity surface emitting lasers," Phys. Rev. A, vol. 63, no. 023 817, Feb. 2001.

[13] C. Degen, B. Krauskopf, G. Jenemann, I. Fischer, and W. Elsäßer, "Polarization selective symmetry breaking in the near-fields of vertical cavity surface emitting lasers," J. Opt. B-Quantum. S. O., vol. 2, pp. 517-525, Aug. 2000
[14] P. Debernardi, G. P. Bava, C. Degen, I. Fischer, and W. Elsäßer, "Influence of anisotropies on transverse modes in oxide-confined VCSEL's," IEEE J. Quantum Electron., vol. 38, pp. 73-84, Jan. 2002.

[15] J. Mulet and S. Balle, "Spatio-temporal modeling of the optical properties of VCSEL's in presence of polarization effects," IEEE J. Quantum Electron., vol. 38, pp. 291-305, Mar. 2002.

[16] A. Valle, L. Pesquera, S. I. Turovets, and J. M. Lopez, "Nonlinear dynamics of current-modulated vertical-cavity surface-emitting lasers," Opt. Commun., vol. 208, pp. 173-182, Jul. 2002.

[17] P. Höß, K. Fleder, and J. Ehrhardt, "Subnanosecond optical gating using coax cable input microchannel plate image intensifier," Opt. Eng., vol. 37, pp. 2213-2216, Aug. 1998.

[18] A. Valle, J. Sarma, and K. A. Shore, "Dynamics of transverse mode competition in vertical cavity surface emitting laser diodes," Opt. Comm., vol. 115, pp. 297-302, Mar. 1995.

[19] O. Buccafusca, J. L. A. Chilla, J. J. Rocca, C. Wilmsen, S. Feld, and R. Leibenguth, "'Ultrahight frequency oscillations and multimode dynamics in vertical cavity surface emitting lasers," Appl. Phys. Lett., vol. 67 , pp. 185-187, July 1995.

[20] A. Valle, J. Sarma, and K. A. Shore, "Spatial holeburning effects on the dynamics of vertical cavity surface-emitting laser diodes," IEEE J. Quantum Electron, vol. 31, pp. 1423-1431, Aug. 1995.

[21] A. Gahl, S. Balle, and M. San Miguel, "Polarization dynamics of optically pumped VCSEL's," IEEE J. Quantum Electron., vol. 35, pp. 342-351, Mar. 1999.

[22] J. Mulet and S. Balle, "Transverse mode dynamics in vertical-cavity surface-emitting lasers: Spatiotemporal versus modal expansion descriptions," Phys. Rev. A., vol. 66, no. 053 802, Nov. 2002.

[23] I. Fischer, O. Hess, W. Elsäßer, and E. Göbel, "Complex spatio-temporal dynamics in the near-field of a broad-area laser," Europhys. Lett., vol. 35, pp. 579-584, Sept. 1996.

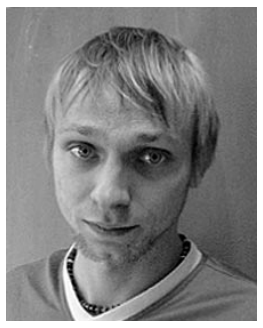

Andreas Barchanski was born in Katowice, Poland, in 1976 . He is currently working toward the diploma degree at Darmstadt University of Technology, Darmstadt, Germany.

His main research interests include spatio-temporal emission properties of VCSELs.

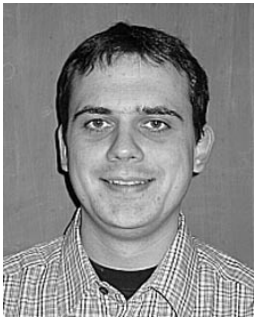

Tobias Gensty was born in Koblenz, Germany, in 1975. He received the diploma degree from Darmstadt University of Technology, Darmstadt, Germany, in 2002, where he is currently working toward the Ph.D. degree.

His main research interests include intensity noise properties of quantum cascade lasers, quantum chaos in VCSELs, and spatio-temporal emission properties of VCSELs.

Christian Degen was born in Neuwied, Germany, in 1970. He received the physics degree in 1997 for work on surface-emitting second-harmonic generation in semiconductor waveguides and the Ph.D. degree in 2001 for experimental work on the mechanisms that determine the transverse mode formation in oxide-confined VCSELs, both from Darmstadt University of Technology, Darmstadt, Germany.

His research interests include spectral, spatial, and polarization effects, as well as quantum-noise properties in semiconductor lasers. Currently, he is a Development Engineer for Infineon Technologies, Munich, Germany, where his attention is focused on the detailed characterization of VCSELs for high-bit-rate optical data transmission. 


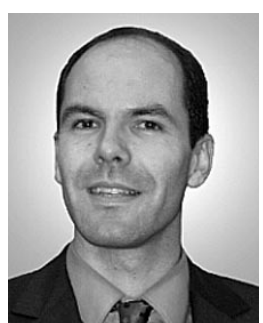

Ingo Fischer was born in Marburg, Germany, in 1966. He received the diploma degree in 1992 and the Ph.D. degree in 1995, both in physics, from Philipps University, Marburg, Germany.

In 1995, he joined the Institute of Applied Physics, Darmstadt University of Technology, Darmstadt, Germany, where his research activities are centered around spatial, spectral, and dynamical emission properties of semiconductor lasers. His studies concentrate on nonlinear dynamics, synchronization of coupled lasers, VCSEL emission, high-power laser emission, phase conjugation, femtosecond spectroscopy, and terahertz generation. His research stays have included AFRL/DELO, Albuquerque, NM, in 1999, and ATR, Kyoto, Japan, in 1999 and 2000.

Dr. Fischer received the Adolf-Messer Foundation Research Prize in 2000.
Wolfgang Elsäßer (M'94-SM'97) was born in Pforzheim, Germany, in 1954. He received the diploma degree in physics from the Technical University of Karlsruhe, Karlsruhe, Germany, in 1980, the Ph.D. degree in physics from the University Stuttgart, Stuttgart, Germany, in 1984, and the Habilitation degree in experimental physics from the Philipps-University Marburg, Marburg, Germany, in 1991.

From 1981 to 1985 , he was with the Max-Planck-Institute for Solid State Research, Stuttgart, Germany. From 1985 to 1995, he was with Philipps-University Marburg. Since 1995, he has been a Full Professor in the Institute for Applied Physics, Technical University of Darmstadt, Darmstadt, Germany. He was on sabbatical leave in 1992 with the Ecole Nationale Superieure des Telecommunications, Paris, France. His research stays have included Trinity College, Dublin, Ireland, in 1990, 1996, 1999, and 2000.

Dr. Elsäßer is a Member of the German Physical Society (DPG). He was awarded the Otto-Hahn-Medal (1985), the Werner-von-Siemens-Medal (1985), the Rudolf-Kaiser Prize (1991), and the IEE J. J. Thomson Premium (1995). 